\title{
Numerical and Experimental Studies on the Chaotic Dynamics of Driven Gunn Oscillator
}

\author{
B. C. Sarkar ${ }^{1 *}$, J. Chakraborty ${ }^{1}$ and S. Sarkar ${ }^{2}$ \\ 'Department of Physics, The University of Burdwan, Golapbag, Burdwan-713104, West Bengal, \\ India; bcsarkar_phy@yahoo.co.in \\ 2Department of Electronics, Burdwan Raj College, Burdwan-713104, West Bengal, India
}

\begin{abstract}
Dynamics of a microwave (X band) Gunn oscillator in presence of a periodic forcing signal has been critically studied to explore the existence of chaos in the behavior of the driven Gunn oscillator (DGO). A broad band continuous spectrum is experimentally observed at the output of the DGO just at the verge of the synchronization band for certain strength and frequency of the forcing signal. For characterisation of the obtained broad band signal, time domain analysis of the frequency down converted DGO output is done and different chaos quantifiers like Maximum Lyapunov exponent and Correlation dimension are evaluated to confirm the chaotic mode of oscillations. Numerical simulation of the system equations also indicate that the dynamics of the DGO enters into a chaotic domain at these conditions.
\end{abstract}

Keywords: Driven System, Gunn Diode, Microwave Chaos, Synchronized Oscillator

\section{Introduction}

The use of solid state microwave Gunn Oscillators (GOs) in various communication-systems is well documented in the literature ${ }^{1-3}$. For the purpose of lock-in amplification, tracking filtering, electronically tunable phase shifting etc., GOs are used in injection synchronized mode. As such, a sizable amount of work has been reported in the last few decades on synchronization characteristics of $\mathrm{GOs}^{4-6}$. In recent years interests are being shown to study the dynamics of the GO under various conditions of operation to explore the possibility of existence of chaotic behaviour in this complex nonlinear system ${ }^{7-9}$. In the present work we revisit the dynamics of a GO driven by an external periodic signal to identify possible chaotic state in it. It has been already reported by the authors that a GO driven by more than one injecting signals becomes chaotic for some specific relative strengths of the driving signals and their frequencies ${ }^{10}$.On the other hand, in this paper we show that a GO driven by a single signal having frequency close to its free running frequency may enter into a chaotic state just before attaining the synchronized state

The paper has been organized in the following way. In Section 2, we formulate the differential equations describing the system under study. For this purpose ac equivalent circuit of the DGO is used. Results of the numerical analysis are presented in section 3 . In section 4 describing the hardware circuit arrangement, results of the experimental studies on DGO are presented. Finally, outcome of the present study and its practical importance are presented in the concluding section 5 .

\section{System Equation of the Driven Gunn Oscillator}

Figure 1(a) shows the functional block diagram of the experimental arrangement of the Gunn oscillator driven by an external signal. To establish the mathematical

${ }^{*}$ Author for correspondence 


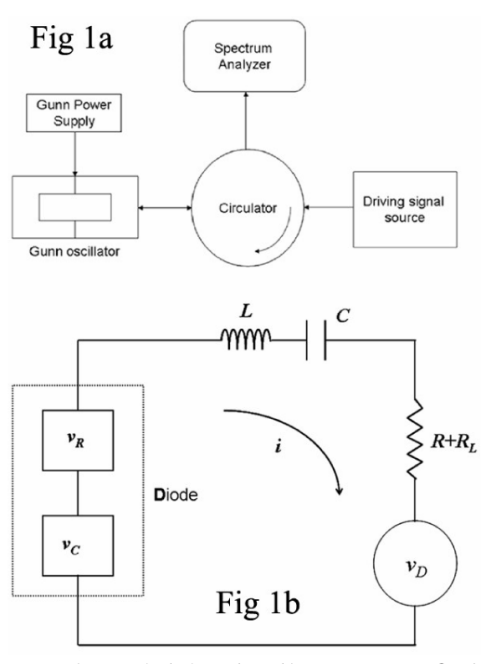

Figure 1. (a) Functional block diagram of the experimental arrangement of the Gunn oscillator driven by an external signal and (b) the series equivalent circuit of it.

equations of the system, we consider its corresponding series equivalent circuit $^{8}$ as shown in Figure 1(b). As a practical Gunn oscillator is designed by mounting a Gunn diode within a waveguide cavity then in the equivalent circuit, Gunn diode is taken as combination of a nonlinear negative resistor $\left(r^{d}\right)$ and a capacitor $\left(c^{d}\right)$ and the cavity is replaced by a series combination of resistor $(R)$, inductor $(L)$ and capacitor $(C)$; $R^{L}$ is the external load connected with it. The effect of the driving signal is taken into account by connecting a voltage source $\left(v^{D}\right)$ in series with the cavity parameters. The device parameters $\left(r^{d}\right.$ and $\left.c^{d}\right)$ are nonlinear in nature. Hence the voltage drops $v^{r}$ across $r^{d}$ and $v^{c}$ across $c^{d}$ would be nonlinear functions of the instantaneous current $\left(i=\frac{d q}{d t}\right)$ and instantaneous charge (q) respectively. Considering up to the cubic nonlinearity term and remembering that the device offers negative resistance, $v^{r}$ and $v^{c}$ are expressed as follows:

$$
\begin{aligned}
& v_{r}=-\beta_{1} i+\beta_{3} i^{3} \\
& v_{c}=-\alpha_{1} q+\alpha_{3} q^{3}
\end{aligned}
$$

Here $\beta_{1}, \beta_{3}$ and $\alpha_{1}, \alpha_{3}$ are the device parameters and their magnitude would depend on the dc bias point. Now applying Kirchhoff's' mesh law in Figure 1(b), one can write the system equation of the driven GO as follows:

$$
L \frac{d i}{d t}+\left(R+R_{L}\right) i+\frac{\int i d t}{C}+v_{r}+v_{c}=v_{D}
$$

Using (1), (2) and considering $v_{D}=A_{d} \cos \left(\omega_{d} t\right)$ (here, $A_{d}$ and $\omega_{d}$ are respectively the amplitude and the angular frequency of the driving signal), (3) can be written in the normalized form as:

$$
\frac{d^{2} q}{d \tau^{2}}=a q-b q^{3}+c \frac{d q}{d \tau}-d\left(\frac{d q}{d \tau}\right)^{3}+\delta \sin \left(\Omega_{d} \tau\right)
$$

Here, we put $\omega_{r}=\frac{1}{\sqrt{L C}}$ (Resonant frequency of the cavity), $\omega_{r} t=\tau$ (Normalized time), $a=\alpha_{1} C-1, b=\alpha_{3} C, c=\left(\beta_{1}-R-\right.$ $\left.R_{L}\right) / \omega_{r} L, d=\beta_{3} \omega_{r} / L, \delta$, and $\Omega_{d}$ represent respectively the charge equivalent to the amplitude of the driving signal and its normalized angular frequency. Using (4) we have studied the dynamics of the GO in presence of an external driving signal. As the parameters $a, b, c, d$ are related with the properties of both the Gunn diode and the wave guide cavity of the GO, so their values should be suitably chosen for stable periodic mode of oscillation in isolated condition. The parameters $a$ and $b$ are related with the device parameters $\alpha^{1}$ and $\alpha^{3}$ (that are associated with the bias current dependent capacitance of the Gunn diode), hence the frequency tuning characteristics of the GO is controlled by them. The parameter $c$ is associated with $\beta^{1}$, the resistance of the active device, so the effect of bias voltage is directly reflected through $c$. The parameter $d$ is related with $\beta^{3}$, the cubic nonlinearity term of the device resistance. Hence, $d$ has the property to check the growth of oscillation amplitude for steady state oscillations. Moreover, since the relative magnitude of $\beta^{3}$ is less than $\beta^{111}$, the value of $d$ should be much less than the parameter $c$.

The analytical expression ${ }^{8}$ of the free running frequency of oscillation $\left(\Omega^{0}\right)$ of the GO can be obtained after substituting $\delta=0$ in (4). It is as follows:

$$
\Omega_{0}^{2}=\frac{a}{2}\left[\left(1+\frac{4 b c}{d a^{2}}\right)^{\frac{1}{2}}-1\right]
$$

\section{Numerical Analysis}

Equation (4) is a non-autonomous nonlinear second order ordinary differential equation (ODE). For numerical simulation of this equation, we decompose it into two first order differential equations as follows:

$$
\frac{d q}{d \tau}=p
$$




$$
\frac{d p}{d \tau}=a q-b q^{3}+c p-d p^{3}+\delta \sin \left(\Omega_{d} \tau\right)
$$

These equations have been solved numerically using fourth order Runge-Kutta technique. The system response is studied by noting the time variation of $q$, phase plane plot of $q$ and $p$ and frequency components present in time varying signal (obtained by first Fourier transform (FFT) of time samples of $q$ ). Physically $q(t)$ is proportional to the GO output. To have the steady state response of the system, a sufficient number of samples of state variables $q$ and $p$ from the initial time have not been taken into account.

At first, dependence of $\Omega^{0}$ on the parameters $a$ and $b$ are examined analytically as well as from simulation of the system equations. Figure 2 and Figure 3 show respectively the variation of $\Omega^{0}$ with $a$ and $b$ respectively. There is a difference between the results as obtained from the numerical simulation and that from the analytical expression. This is due to the fact that the analytical result is based on an assumed sinusoidal solution of the system equation and the simulated results are based on numerical integration that provides a nearly sinusoidal solution in general. Further the analytical method consider a nearly time independent amplitude which is somewhat approximate. The values of the parameters $a$ and $b$ are suitably chosen so that simulated and analytical results are in close agreement. In our present work, oscillator parameters are selected as $a=1.0, b=1.0, c=0.05$ and $d=0.015$. In this condition, free running frequency $\left(\Omega^{0}\right)$ and amplitude $\left(A^{0}\right)$ of the GO are obtained as 1.2771 and 1.995 respectively.
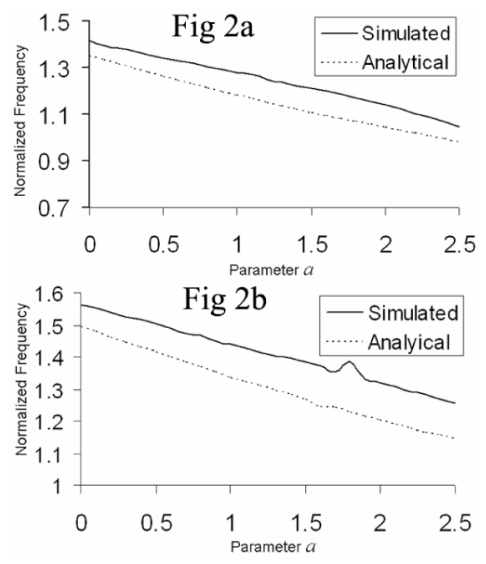

Figure 2. Variation of the normalized angular frequency of free running GO with the parameter $a$ for two different values of the parameter $b[\mathrm{c}=0.05, \mathrm{~d}=.015]$ : (a) $b=1.0$ and for (b) $b=1.5$.
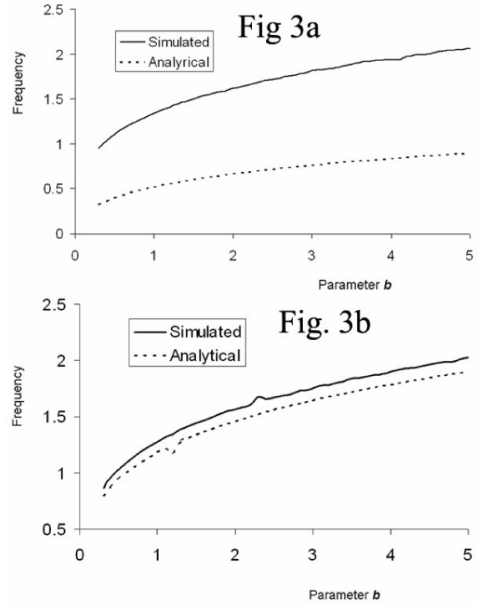

Figure 3. Variation of the normalized angular frequency of free running GO with the parameter $b$ for two different values of the parameter $a[\mathrm{c}=0.05, \mathrm{~d}=0.015]$ : (a) $a=0.5$ and (b) $a=1.0$.

Now, the dynamics of the driven GO is critically examined for a particular strength of the driving signal $(\delta)$ (about $20 \%$ of the strength of the free running GO) and varying its frequency $\left(\Omega^{d}\right)$ gradually from low to a high value, such that the driven oscillator passes through asynchronous mode and then enters into the synchronized state. Crossing this state of synchronization, it again enters into asynchronous 'out of lock' state at higher frequency side. This process is repeated for different values of $\delta$. Some of the obtained results are depicted in Figure 4 and Figure 5 respectively. They include the frequency spectrum of the state variable $p$ (Figure 4 ) and the statespace trajectories in $q$ - $p$ plane (Figure 5). Figure 4 indicates as $\Omega^{d}$ gradually approaches towards the synchronization band of the GO, its output frequency spectrum shows the presence of multiple signal components (Figure 4(a)) having frequencies related to the frequency difference between the GO and the driving signal. The phase plane diagram (Figure 5(a)) indicates the quasi periodic state of the system dynamics. As the external signal frequency is closer to the free running frequency of the $\mathrm{GO}$, the output spectrum is continuous and broad (Figure 4(b)) indicating chaotic oscillations and the phase plane diagram shows a double scroll nature (Figure 5(b)). The synchronized state of the system response is shown in Figures 4(c) and 5(c) respectively. In this situation, the frequency of the GO becomes same as that of the external signal even though it is different from the free running frequency of the GO (Figure 4(c)). The phase plane plot is a closed 
curve (Figure 5(c)) indicating a periodic state. These observations lead to the conclusion that before attaining the synchronized state, a driven GO may jump into a chaotic state of oscillations. As $\Omega^{d}$ is further increased, quasi periodic state of oscillation at the GO output is observed. But no such broad band spectrum as is observed in the lower side of the synchronization band (SB) is observed (Figure 4(d) and Figure 5(d) respectively). Moreover, Figure 6 indicates that as the amplitude of the driving signal enhances probability of chaoticity increases in
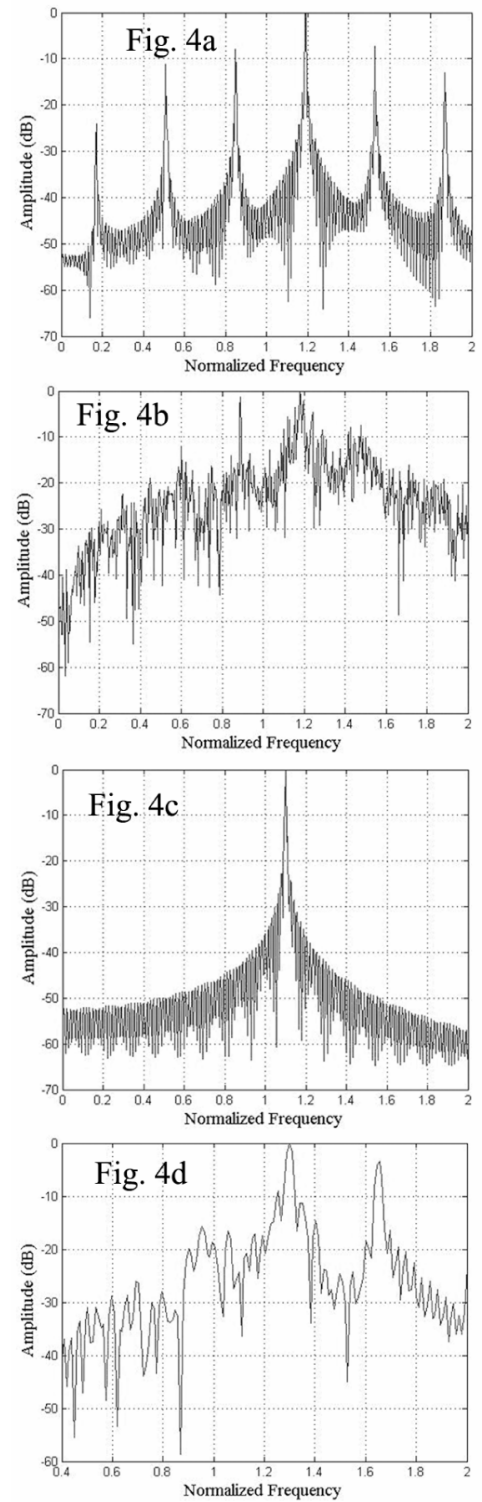

Figure 4. Numerically obtained frequency spectrum of the driven GO for a forcing signal of amplitude $\delta=0.4$ and frequencies (a) $\Omega^{d}=0.85$, (b) $\Omega^{d}=0.89$, (c) $\Omega^{d}=1.1$, (d) $\Omega^{d}=1.65$. The GO parameters are: $a=1, b=1, c=0.05$, $d=0.015$. the verge of the synchronization band at its lower side Table 1 reports the numerically obtained SB of the GO for different amplitudes of the driving signal. As reported elsewhere it shows the increase in the SB with the increase of the driving signal strength ${ }^{6}$.

As an additional proof of this chaotic transition, we have evaluated the Lyapunov exponents (LEs) from the time series data obtained from the state equations of the
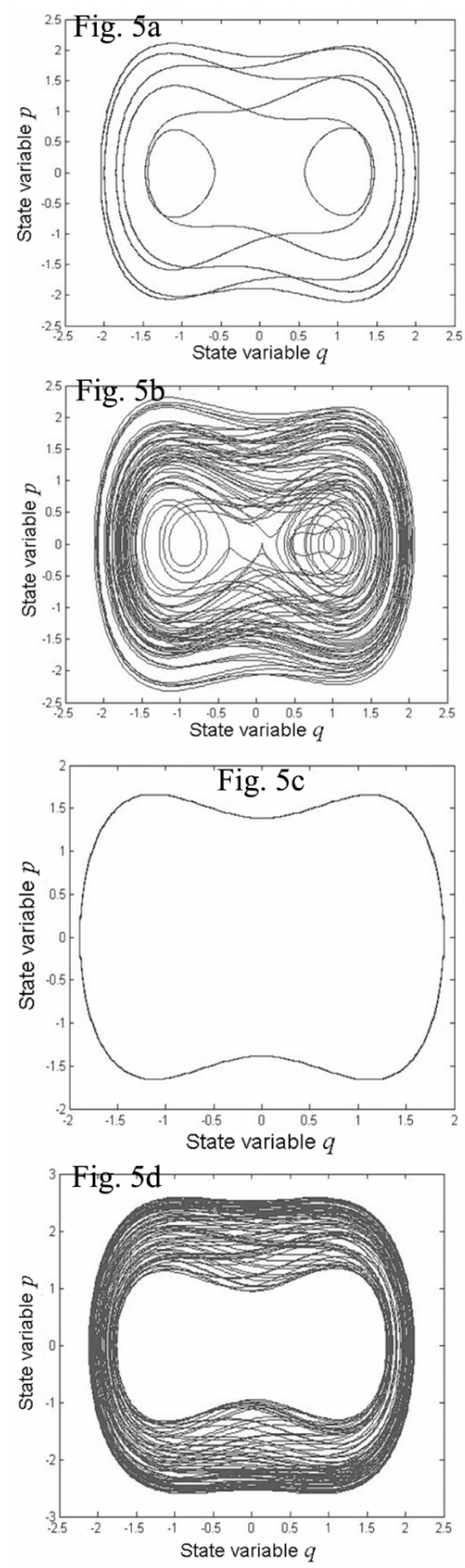

Figure 5. Numerically obtained phase plane plot of $q-p$ of the driven GO for different frequency of the forcing signal [ $\delta$ $\left.=0.4, A^{0}=1.995, \Omega^{0}=1.2771\right]$ (a) $\Omega^{d}=0.85$, (b) $\Omega^{d}=0.89$, (c) $\Omega^{d}=1.1,(\mathrm{~d}) \Omega^{d}=1.65$. 
Table 1. Numerically obtained SB of the GO for different driving signal amplitudes (GO parameters: $a=1, b=1, c=0.05, d=0.015$, $\left.\Omega_{0}=1.2771, A^{0}=1.995\right)$

\begin{tabular}{cccc}
\hline $\begin{array}{c}\text { Driving signal } \\
\text { amplitude normalized } \\
\text { to the free-running } \\
\text { oscillator amplitude }\end{array}$ & $\begin{array}{c}\text { Lower side } \\
\text { frequency } \\
\text { limit of the SB } \\
\text { (normalized) }\end{array}$ & $\begin{array}{c}\text { Upper side } \\
\text { frequency } \\
\text { limit of the SB } \\
\text { (normalized) }\end{array}$ & $\begin{array}{c}\text { Synchronization } \\
\text { bandwidth (SB) } \\
\text { (normalized) }\end{array}$ \\
\hline 0.20 & 1.04 & 1.49 & 0.45 \\
0.30 & 0.95 & 1.55 & 0.60 \\
0.40 & 0.94 & 1.61 & 0.67 \\
0.50 & 0.93 & 1.67 & 0.74 \\
0.60 & 0.92 & 1.70 & 0.78 \\
\hline
\end{tabular}

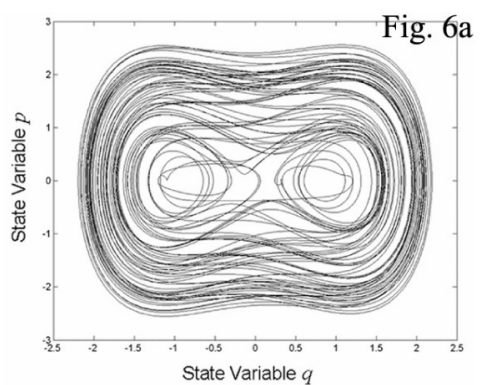

Fig. $6 \mathrm{~b}$

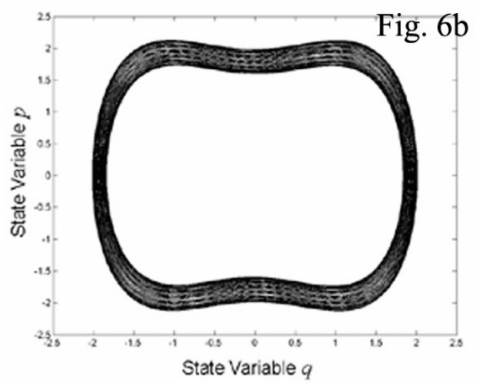

Figure 6. Numerically obtained phase plane plot of the driven $\mathrm{GO}$ for the forcing signal frequency $\Omega^{d}=0.89$ and two different amplitudes: (a) $\delta=0.4$, (b) $\delta=0.2$. The parameters of the GO is $\left[\Omega^{0}=1.2771, A^{0}=1.995\right]$.

DGO ((6) and (7)). For this purpose the algorithm proposed by Wolf et al. ${ }^{12}$ is used. The nature of variation of the maximum Lyapunov exponent (MLE) with the driving signal parameters $\delta$ and $\Omega^{d}$ is shown in Figure 7. The dynamics of the system can be easily understood from an examination of the MLE. It has been observed MLE becomes positive for certain parameter zone of $\delta$ and $\Omega^{d}$ which indicates transition to chaotic state of the driven GO.

\section{Experimental Studies}

In the experimental arrangement as shown in Figure 1, the GO under study is a commercial available one (Vidyut

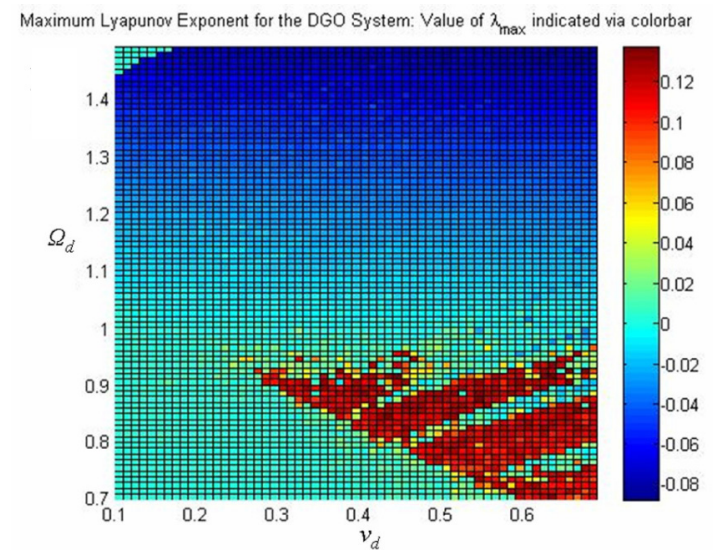

Figure 7. Variation of the Maximum Lyapunov exponent as calculated from the numerically obtained time series data of the system equations of the driven GO $[a=1.0, b=1.0$, $c=0.05, d=0.015$.

Yantra Udyog, X2152). It is driven by a periodic signal obtained from a microwave signal generator (Agilent Technologies, N5183A). For coupling the GO with the driving source we have used a circulator (SICO, XC621) whose port-1 and port- 2 are connected with the driving source (DS) and the GO respectively. Port- 3 of the circulator is used for observation of the GO dynamics.

In free running condition, bias voltage of the GO is adjusted at 9.57 volts that gives stable periodic oscillation having frequency $f^{0}=10 \mathrm{GHz}$ and output power $P^{0}=13 \mathrm{dBm}$. In this condition DS is applied into the oscillator. Keeping power $\left(P^{d}\right)$ of the injected signal fixed, its frequency $\left(f^{d}\right)$ is gradually varied from low to a high value, such that the driven oscillator passes through asynchronous mode and then enters into synchronized state. Crossing this state of synchronization, it again enters into asynchronous 'out-of-lock' state at higher frequency side. During this process, the GO dynamics 
is critically examined. This study is repeated for different power of the DS.

\subsection{Observation in Microwave Frequency Range}

For experimental observation of the driven GO dynamics directly, a Spectrum Analyser (SA)( Rhode and Schwartz, FSL-18) is connected with the port 3 of the circulator as depicted in Figure 1. Outcome of the experimental observations are described below:

(i) When $f^{d}$ is far from the synchronization band SB and at the lower side of it, there are some windows in the frequency span of the DS where a number of complex behaviours like quasi periodic nature of oscillation and random variation in its amplitude showing broad band continuous spectrum are observed. The quasi periodic state occurs when the ratio between $f^{0}$ and $f^{d}$ is an irrational number. However, adjusting the strength and the frequency of the DS, apparent random behaviour can be observed in the GO dynamics. This suggests that in this condition chaotic state of oscillation can be obtained from the GO.

(ii) As $f^{d}$ is further increased, the GO enters in stable synchronized mode of operation and its output shows single frequency spectrum.

(iii) With further increase in $f^{d}$, the oscillator exits from its synchronized domain. Interestingly, in this frequency domain (ie 'out-of-lock' condition) only quasi periodic nature of oscillation is observed. It indicates, in the upper side of its $\mathrm{SB}$, no such random variation in the output amplitude of the GO is possible.

(iv) With the increase of the strength of DS, both the SB of the GO and the range of $f^{d}$ for which broad band continuous natured spectrum at the lower side of the SB as observed are enhanced. Moreover, width of the generated broad band spectrum is also increased as strength of the DS is increased.

(v) Required strength of the DS for apparent random variation in the output amplitude of the GO (in asynchronous mode operation at the lower side of SB) increases with the decrease in its frequency detuning with respect to the GO. However, bandwidth of the generated broad band spectrum increases with the strength of the DS.

The observed experimental results are summarized in Table 2 and Table 3. Some of the observed output spectra of the driven GO with variation of frequency and strength of the DS respectively are presented in Figure 8 and Figure 9.

\subsection{Observations in Frequency Down Converted Condition}

To characterise the broad band signal as obtained from the DGO output, time domain analysis using real time oscilloscope is required. But, due to non availability of oscilloscope in X-band frequency range, we have taken an indirect method based on frequency down conversion principle. Figure 10 shows the schematic diagram

Table 2. Characteristics of the driven GO for different strength and frequency of the driving signal

\begin{tabular}{ccc}
\hline $\begin{array}{c}\text { Relative power } \\
\text { of the diving } \\
\text { signal }\left(P^{d} / P^{0}\right)\end{array}$ & $\begin{array}{c}\text { Frequency of the } \\
\text { driving signal } \\
\left(f^{d}\right) \text { in GHz }\end{array}$ & $\begin{array}{c}\text { Nature of the } \\
\text { dynamical state of the } \\
\text { DGO }\end{array}$ \\
\hline 0.25 & $\begin{array}{c}9.973-9.977 \\
9.978-10.016\end{array}$ & $\begin{array}{c}\text { Quasi periodic } \\
\text { Broadband Continuous }\end{array}$ \\
& $\begin{array}{c}\text { Synchronized } \\
\text { (SB=38 MHz) }\end{array}$ \\
& $9.900-9.966$ & Quasi periodic \\
$9.967-9.975$ & Quasi periodic \\
$9.976-10.017$ & $\begin{array}{c}\text { Synchronized } \\
\text { (SB=41 MHz) }\end{array}$ \\
& $10.018-10.100$ & Quasi periodic \\
& $9.900-9.960$ & Quasi periodic \\
$9.961-9.969$ & Broadband Continuous \\
$9.970-10.021$ & Synchronized \\
& & (SB=51 MHz) \\
& & Quasi periodic \\
\hline
\end{tabular}

Table 3. Required strength of the driving signal for different frequency to occur broad band spectrum and measured $30 \mathrm{dBm}$ bandwidth of this spectrum

\begin{tabular}{ccc}
\hline $\begin{array}{c}\text { Frequency of the } \\
\text { driving signal } \\
\left(f^{d}\right) \text { in GHz }\end{array}$ & $\begin{array}{c}\text { Required power of } \\
\text { the driving signal for } \\
\text { occurrence of broad } \\
\text { band spectrum }(\mathrm{dBm})\end{array}$ & $\begin{array}{c}30 \mathrm{dBm} \\
\text { bandwidth of } \\
\text { broad band } \\
\text { spectrum } \\
(\mathrm{MHz})\end{array}$ \\
\hline 9.980 & 3.0 & 25.0 \\
9.975 & 5.11 & 40.0 \\
9.970 & 6.34 & 50.0 \\
\hline
\end{tabular}




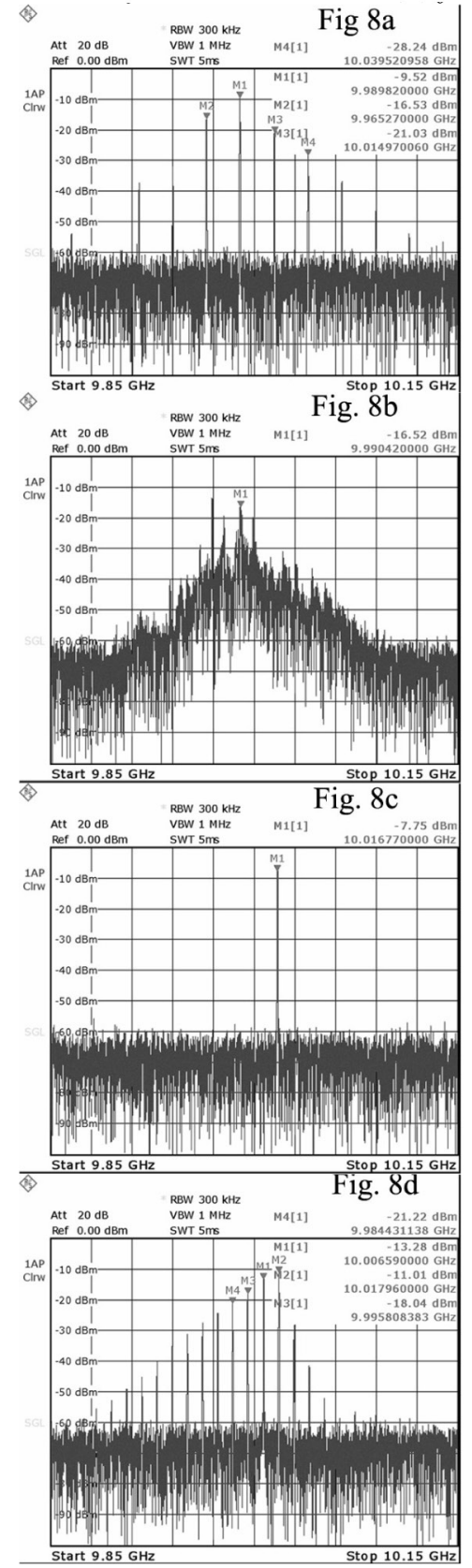

Figure 8. Experimentally obtained spectral characteristic of the driven $\mathrm{GO}$ for different frequencies of the forcing signal with fixed relative power $P^{d} / P^{0}=0.28$ (a) $f^{d}=9.965 \mathrm{GHz}$, (b) $f^{d}=9.969 \mathrm{GHz},(\mathrm{c}) f^{d}=10.017 \mathrm{GHz},(\mathrm{d}) f^{d}=10.018 \mathrm{GHz}$.

of the hardware arrangement used for this purpose. It is designed using a magic tee (SICO, XE345) and two diode detectors (SICO, XD451). Output $\left(E^{1}(t)\right)$ of the driven $\mathrm{GO}$ is applied at the E-arm and a fixed frequency reference signal $(10.3 \mathrm{GHz})\left(E^{2}(t)\right)$, obtained from another $\mathrm{GO}$ (Vidyut Yantra Udyog, X2152) is applied at the $\mathrm{H}$-arm of the magic tee. Then, sum and difference of $E^{1}(t)$ and $E^{2}(t)$

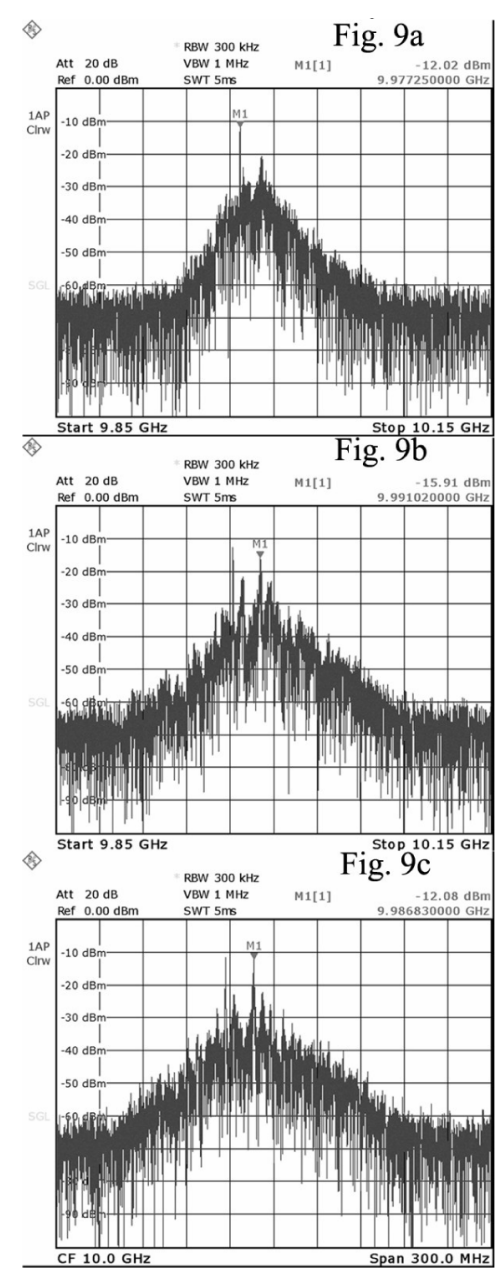

Figure 9. Variationin thebroadbandspectralcharacteristics of the driven GO for different relative powers of the driving signal (a) $P^{d} / P^{0}=0.25, f^{d}=9.977 \mathrm{GH}$, (b) $P^{d} / P^{0}=0.28$, $f^{d}=9.972 \mathrm{GHz}$, (c) $P^{d} / P^{0}=0.35, f^{d}=9.967 \mathrm{GHz}$.

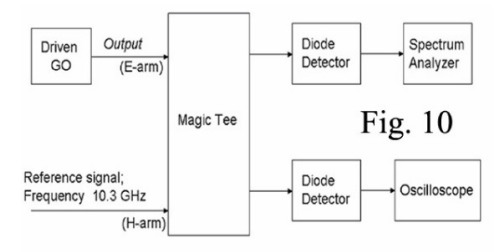

Figure 10. Schematic diagram of the frequency down conversion scheme.

respectively would be obtained from its other two terminals. If now, diode detectors are connected with each of these output terminals of the magic tee, we would obtain Frequency Down Converted (FDC) signals in $\mathrm{MHz}$ frequency range (equal to the difference in frequencies as obtained from the driven GO output and the reference signal) from these two detectors. One of these signals is 
used in a SA (Agilient Technologies, N9010A) to examine the spectral nature of the FDC signal. The other one is applied in a storage oscilloscope (Agilient Technologies, Infinium 54810 A) for capturing the time series data. These data are then analysed using the commercial chaos data analyser CDA by Sprott ${ }^{13}$ and chaos quantifiers like Maximum Lyapunov Exponent (MLE) and Correlation Dimension (CD) are evaluated. Figure 11 and Figure 12 show respectively the nature of variation in the spectral characteristics of the FDC signal with the variation of the DS frequency and strength. Table 4 gives an estimation of the calculated values of MLE , CD and $30 \mathrm{dBm}$ bandwidth of the FDC signal where broad band continuous

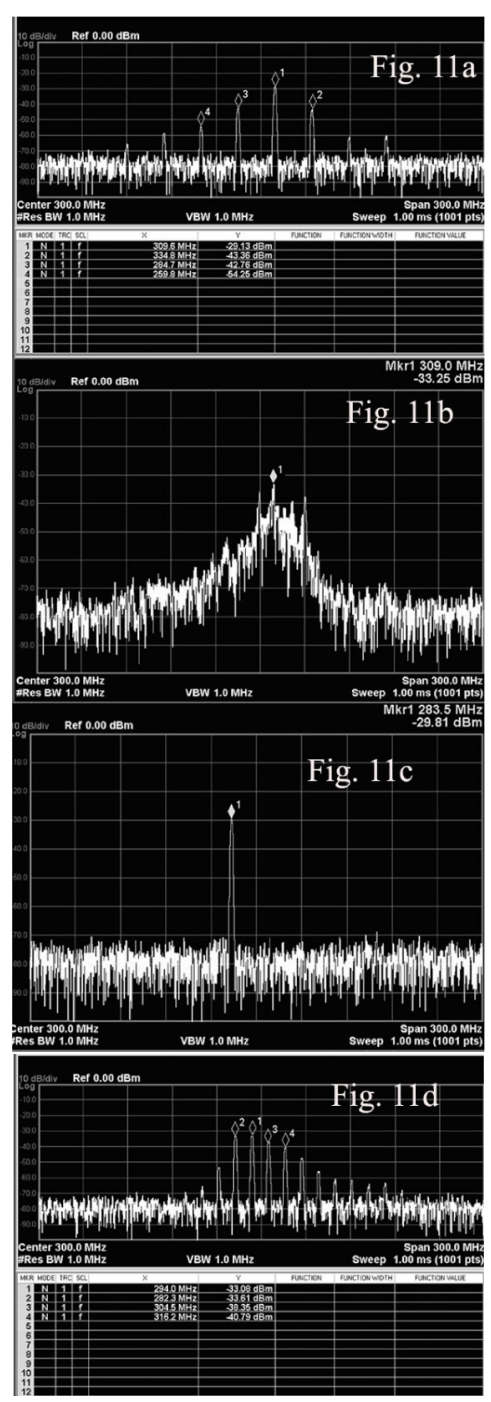

Figure 11. Variation in the spectral characteristic of the FDC signal for different strength of the driving signal $\left(\left(P^{d} / P^{0}=0.28\right)\left(\right.\right.$ a) $f^{d}=9.965 \mathrm{GHz}$, (b) $f^{d}=9.969 \mathrm{GHz}$, (c) $f^{d}=10.017 \mathrm{GHz}$, (d) $f^{d}=10.018 \mathrm{GHz}$. spectrum is observed. Positive value of MLE and non integer value of $C D$ are obtained in this condition. It indicates that by adjusting the strength and the frequency of the DS, it is possible to transit the dynamics of the driven $\mathrm{GO}$ to chaotic state. Comparing Figure 8 with Figure 11 and Figure 9 with Figure 12, we have observed the nature of variation in the spectral characteristics of the driven GO output and their FDC parts are identical.

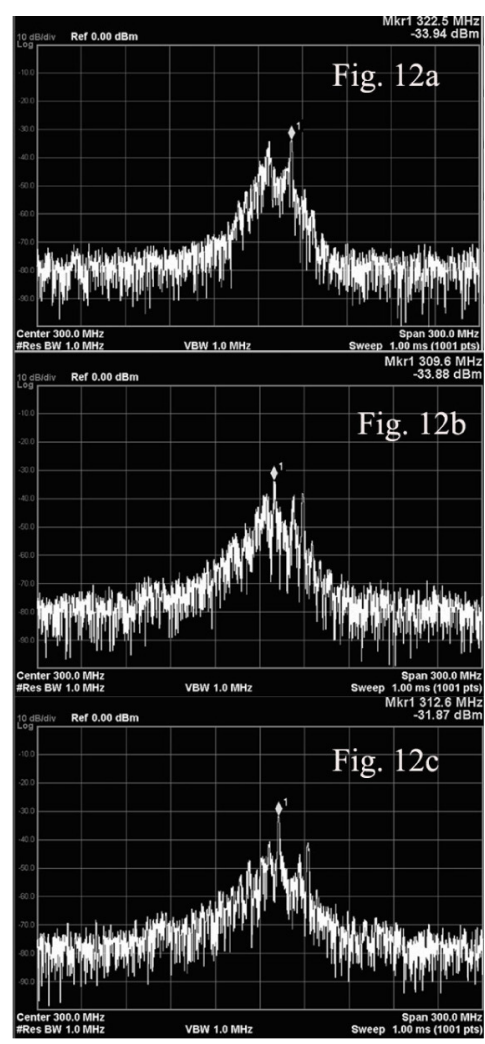

Figure 12. Variation in the broad band spectral characteristics of the FDC signal for different strength of the driving signal (a) $P^{d} / P^{0}=0.25, f^{d}=9.977 \mathrm{GH}$, (b) $P^{d} / P^{0}=0.28$, $f^{d}=9.972 \mathrm{GHz}$, (c) $P^{d} / P^{0}=0.35, f^{d}=9.967 \mathrm{GHz}$.

Table 4. MLE, CD and $30 \mathrm{dBm}$ band width of the chaotic spectrum for different frequency and strength of the driving signal

\begin{tabular}{ccccc}
\hline $\begin{array}{c}\text { Relative } \\
\text { power of } \\
\text { the diving } \\
\text { signal }\left(\boldsymbol{P}^{d} / \boldsymbol{P}^{0}\right)\end{array}$ & $\begin{array}{c}\text { Frequency } \\
\text { of the } \\
\text { driving } \\
\text { signal }\left(\boldsymbol{f}^{d}\right) \\
\text { in } \mathrm{GHz}\end{array}$ & $\begin{array}{c}\text { Measured } \\
\mathbf{3 0 \mathrm { dBm }} \\
\text { bandwidth of } \\
\text { the chaotic } \\
\text { spectrum }\end{array}$ & MLE & CD \\
\hline 0.25 & 9.977 & $50 \mathrm{MHz}$ & 0.424 & 3.687 \\
0.28 & 9.972 & $40 \mathrm{MHz}$ & 0.392 & 3.836 \\
0.35 & 9.964 & $35 \mathrm{MHz}$ & 0.311 & 3.754 \\
\hline
\end{tabular}




\section{Conclusion}

The dynamics of a driven GO is extensively studied through numerical simulation as well as experimental methods. This study confirms the occurrence of synchronization in a driven GO for a proper set of system and driving signal parameters .Besides this, the study has reveal a region of chaotic oscillation of the driven GO. This state appears when the driving signal frequency is slowly increased from the lower side of the so called synchronization band of the system. All the numerical and experimental studies qualitatively confirm the existence of chaotic regime in the driven oscillator dynamics. As such a GO driven by a suitable external signal can be used to generate chaotic oscillation of microwave frequency predictably. In this direction this study reported in this paper has practical importance along with an academic interest.

\section{Acknowledgement}

Authors acknowledge partial financial assistance from DST (India) and DAE, BRNS (India) through sponsored research projects in carrying out the work.

\section{References}

1. Alekseev YI. Determination of basic characteristics of synchronization of Gunn self-excited oscillators. Instrum Exp Tech. 2007; 50(2):241-43.

2. Bates RS, Feeney S. Novel varactor tuned millimeter wave Gunn oscillator. Electron Lett. 1987; 23(14):714-15.
3. Takayama Y. Doppler signal detection with negative-resistance diode oscillators. IEEE Trans Microw Theor Tech. 1973; 21(2):89-94.

4. Kurokawa K. Injection locking of microwave solid state oscillator. IEEE Proceedings. 1973; 61:1386-410.

5. Biswas BN, Chatterjee S, Sarkar S, Bhattacharyay AK, Ray SK. Bias tuned injection locked discriminators. IEEE Trans Microw Theor Tech. 1987; 35(9):812-18.

6. Sarkar S. Structure and application of a modified lock-in notch filter using solid state microwave oscillator. 1994; $1: 149-52$.

7. Sarkar BC, Koley C, Guin AK, Sarkar S. Some numerical and experimental observations on the growth of oscillations in an X-band Gunn oscillator. Progress In Electromagnetics Research B. 2012; 40:325-41.

8. Sarkar BC, Sarkar D, Sarkar S, Chakravorty J. Studies on the dynamics of bilaterally coupled X-band gunn oscillators. Progress in Electromagnetics Research B. 2011; 32:149-67.

9. Jongfu. J, Benkun M. Period doubling and chaos in the Gunn effect. Phys Rev B. 1991; 44(20):72-4.

10. Chakravorty J, Banerjee T, Ghatak R, Bose A, Sarkar BC. Generating chaos in injection-synchronized Gunn Oscillator: An experimental approach. IETE Journal of Research. 2009; 55:106-11.

11. Gonorovsky IS. Radio Circuits and Signals. Moscow-Mir Publishers, (Chapter 8); 1981.

12. Wolf A, Swift JB, Swinney HL, Vastano JA. Determining Lyapunov exponents from a time series. Physica D. 1985; 16:285-317.

13. Sprott JC. Chaos Data Analyzer Package. Available from: http://sport.physics.wise.edu/cda.htm 